\title{
WHAT DOES COMPUTER SUPPORT FOR COOPERATIVE WORK MEAN? A STRUCTURATIONAL ANALYSIS OF COMPUTER SUPPORTED COOPERATIVE WORK
}

\author{
Kalle J. Lyytinen \\ University of Jyväskylä \\ Ojelanki K. Ngwenyama \\ University of Michigan
}

\begin{abstract}
Technical developments in electronic communication and computing coupled with new understanding of relationships between computers and work processes has given impetus to a significant amount of research in the area of Computer Supported Cooperative Work (CSCW). Much of this work, however, lacks strong theoretical foundations, and there is no clear definition of CSCW, the major research questions of the field, or appropriate strategies for research. In this paper we suggest Giddens" theory of structuration as a conceptual foundation for CSCW research and propose a formal definition for CSCW. We conclude by discussing seven implications of the framework for future research into: (a) platform software features, (b) research methods, (c) systems development approaches, (d) features of CSCW development methodologies, (e) interactions between CSCW use processes and organizational structures, (f) interactions between work processes and CSCW applications, and (g) larger social change due to adoption of $\mathrm{CSCW}$ applications.
\end{abstract}

Keywords: Computer supported cooperative work, computer impact, structuration theory, application features, design.

\section{INTRODUCTION}

Research into Computer Supported Cooperative Work (CSCW) has gained considerable momentum since the mid 1980s. A growing number of new information technology applications often referred to as "groupware" have given impetus to this field (Greif, 1988; Johansen, 1988; Kraemer \& King, 1988). Research in this area has been loosely defined in the literature as the study of the design, delivery, and evaluation of CSCW applications. The motivation for this definition stems from the fact that these applications generally combine communicating, computing, and other technologies to facilitate cooperation, coordination, or decision-making by a group of people. This research spans a heterogeneous set of topics, from studies of organizational impact of electronic mail, to implementations of network protocols. As Howard (1987) ironically points out, the only common denominator among researchers in this area is their belief in the goodness of the "cooperation."

Although the term CSCW hints at more "socially" oriented applications, there have been few attempts (cf. Sörgaard, 1988; Suchmann, 1989; Robinson, 1989; Bannon \& Schmidt, 
1990), to carefully define the field based on a sociological articulation of: (a) What "cooperation" and its "computer support" could mean? (b) What specific features of CSCW applications provide the target and profile for research and design? Accordingly, the research agenda is largely technology driven, fragmented, and lacking clear theoretical focus and direction. A significant amount of foundational work needs to be done in order to define a general research agenda for the field. As Lyytinen (1991) noted, the current state of CSCW research warrants the formulation of a new research agenda to examine computer mediated group-level interactions in social milieux. There are several questions that must be addressed in order to build up such an agenda. These questions are: (a) What constitutes CSCW applications, that is, what is the nature (ontology) of cooperative work and what do we mean by its computer support? (b) What is an appropriate theoretical framework for apprehending the intricate issues of the CSCW problem space? (c) What are the major research problems suggested by the theoretical framework? and (d) What research methods are appropriate for enquiring into this problem space?

In this paper we address these issues. First, we outline a theoretical framework, based on Giddens' theory of structuration (Giddens, 1979, 1984), upon which properties of CSCW applications can be described and analyzed. We believe that the interactions between cooperative work and computer support can be more clearly explicated from this theoretical perspective. Second, we demonstrate the value of the theoretical framework by using it to develop a normative definition of CSCW applications and to distinguish three desirable features of these applications that we believe to be of fundamental importance in their development and research. Based on this conceptual analysis we identify a number of design and research issues that need to be addressed in future research. The paper aims to build a foundation upon which observations and assessments in future research into CSCW can be grounded.

\section{MOTIVATION FOR A NEW FRAMEWORK}

The focus in CSCW on interactions between work processes and their computer support presupposes a rich theory of work. However, the traditional view of work which underpins much of the research in computing and information systems is founded on a mechanistic "theory of work." It suggests that work can be divided into a sequence of tasks characterized solely in terms of their "uncertainty" and the need for "information" to make "decisions" (Galbraith, 1977). This view ignores the multidimensional nature and social richness in the articulation of work processes (Mohrman \& Lawler, 1984). It also leads to a simplistic understanding of the role of communications in work processes, and a solely rationalistic explanation of how information systems are used in organizations (Feldman \& March, 1981). Consequently, many information technology (IT) applications conceived from this perspective exhibit Tayloristic work designs, focusing on individual's task productivity while underestimating the importance of the social context. This often leads to inappropriate application designs, difficulty of usage, and outright failure of many systems (Sheil, 1983; Grudin, 1988).

The "received theory" perspective, which has dominated the discourse on information systems research, is too limited for $\mathrm{CSCW}$ research because it does not support a broad 
enough ontological definition of work-technology interactions. There are several other schools of thought that offer richer theoretical foundations from which we could develop alternative conceptualizations of work-technology interactions. One such approach is the "structuration theory" of Anthony Giddens, which provides an "ontological framework for the study of human social activities, that is, recurrent social practices and their transformations" (Giddens, 1984). It offers a high level conceptual scheme that enables researchers to understand: (a) how human agency creates social structures, while at the same time it is constrained by them; and (b) the delicate linkages of reflexively organized action and institutional constraints. One of the principal aims of structuration theory is to resolve the agency/structure debates between interpretivism and functionalism. In the former the emphasis is placed on the way that human agents and their interpretations create the social world. In the latter the emphasis is on the pre-existent, given nature of the objects and structures of social systems.

More recently a small number of researchers have adapted a structuration theory perspective for studying the relationship between information technology and human action. Poole \& DeSanctis (1989) used structuration to examine the use of group decision support systems in organizations. Orlikowski $(1988,1989)$ studied the relationship between software productivity tools and software development practices in a large multinational software firm from a structurational perspective. She also gives an interesting account of the use of structuration theory in understanding the nature of technology in organizations (Orlikowski, 1990). Han \& Walsham (1989) conducted a multilevel structurational analysis of the effect of computerization on public policies in Malaysia. They explored the relationships between computerization actions and social structures at various levels of society. Other researchers have also suggested the use of structuration theory for studying end-user computing (Robey \& Zmud, 1990), information system failures (Lyytinen \& Hirschheim, 1987), systems development and IS strategy (Walsham \& Han, 1990). Applications of structuration theory to other areas of organization science also suggest the breadth of its framework. Some examples are: organization culture (Riley, 1983), accounting (Boland, 1985, Macintosh \& Scapens, 1990) and work organization and technology impact (Barley, 1986).

\section{KEY CONCEPTS OF STRUCTURATION THEORY}

Structuration theory views agency and structure as a duality in which human agents draw upon understandings of interpretive schemas, norms, and power during social action, and in so doing, produce and reproduce social structure. It does not consider them as independently given sets of phenomena as the agency/structure debate assumes. Accordingly, social structures do not exist in a "concretc" sensc, thcy are instantiations of social actions over time intervals. In this way the agency/structure dichotomy is resolved in structuration theory into a duality. This fundamental notion implies that all social activity, including work processes, can be viewed as enabled and constrained by social structures that are continually produced and reproduced via human agency.

Agency refers to the volitional character of human actions; that is, the capability of individuals to act with conscious intention (Giddens, 1984, p. 375). This implies that human beings are knowledgeable agents who can and do monitor the domains of social actions within which they operate. In particular they monitor their own actions and their conse- 
quences, the actions of others, and also other aspects of the domain of action. Identified with this ability for monitoring the domain of action are two levels of consciousness: practical and discursive. Practical consciousness is the capability to maintain a continuing "theoretical understanding" of the grounds of their social activity. Human agency thus exhibits what Giddens calls the rationalization of action. This is, however, not the same as giving rationally valid reasons for particular items of conduct, nor even the capability to do sobut just the agent's knowing what he/she does. People know usually more than what they can say. Discursive consciousness, on the other hand, is reflexive, focusing on the "monitoring of that monitoring of action"; it is the capability to explicitly describe intentions behind actions, the reasons and motivation for action (action potential). These two levels of consciousness are driven by motivations located in the agent's unconscious that basically aims to find psychological security for the agent. This need can largely explain why agents routinely reproduce social terms that they might even recognize as excessively coercive.

In the study of structuration the second part of the duality-structure is given equal emphasis. In Giddens' terms structure consists of "generative rules and resources that members draw upon, but also thereby, change in their production of society" (Giddens, 1976). Thus, structure does not refer to the descriptive analysis of patterns of interaction that normally compose structure in the functionalistic analysis. In Giddens' terms such a surface description is called the systems of social life, or the patterning of social relations across time and space, understood as reproduced practices. From this perspective, structurational analysis focuses on the deeply seated elements of social existence that are recursively employed and instantiated in social practices. These structural features are considered virtual in structuration theory; they are conceptualized as the structural "properties" of (social) systems, that is, sets of rules and resources that are drawn upon and reproduced in action. Accordingly, social structure can only be analyzed by examining the sets of rules invoked and resources deployed by agents within the domain of social interaction. From this perspective it is also not meaningful to talk of a work process that does not involve "cooperative" or social aspects, as it always contains rules and resources that exist only in a social space, such as rules of grammar, idioms of language, standard operating procedures, authoritative structures, typing instructions, manuscript styles, accounting procedures, conventions of financial analysis, etc.

From the structuration theory perspective, rules are techniques or generalizable procedures applied in the production/reproduction of social practices. This definition includes communication codes and linguistic rules, technical directives, valid organizational norms and other rules drawn upon in social interactions. These rules may be codified and articulated as a policy and bureaucratic rules, or exist as unarticulated background knowledge such as rules of grammar-sedimented interpretations of rules rather than rules as such. Resources signify capacities to generate command over material and social objects, which is to generate power. Resources include capacities to affect material objects and means (allocative resources), as well as nonmaterial capacities to harness the activities of other human beings (authoritative resources). As dualities, these social structures constitute both the medium and the outcome of interactions. They form its medium because they provide the rules and resources individuals must draw on to interact meaningfully. They produce its outcome because rules and resources exist only through being applied and acknowledged by the agents - they have no existence independent of the social practices they constitute.

Giddens points out the importance of analyzing social structuring from three dimensions: 
signification, domination, and legitimation. These form the three fundamental elements of social interaction: (a) the constitution of meaning, (b) relations of power, and (c) moral order. These fundamental dimensions of social structure are further illustrated by a schematic chart in Figure 1.

In this diagram, both the social structure and human interaction - the two poles of the duality - are broken down into three dimensions and are interlinked by three modalities as shown. These modalities are drawn upon by actors in the reproduction of social order and relate the capacities of agents into properties of social structure. Modalities may be defined as modes of mediating interaction and structure. As actors mediate action situations they draw upon stocks of implicit background, and explicit foreground knowledge, as well as material and nonmaterial resources. Implicit background knowledge is comprised of interpretative schemes built up from past experiences, socialization, and tradition. Communication, for example, involves the use of shared interpretive schemes that are stocks of knowledge that human actors use to make sense of their and other's communicative actions. This type of knowledge is not necessarily articulable by competent speakers. Nonetheless, used in everyday interactions, this type of knowledge mediates the production and reproduction of meaning or signification. Explicit foreground knowledge is comprised of policies, explicit social norms (codes of conduct, etc.), procedures and rules of action that actors follow in the process of social production and reproduction. Material and nonmaterial resources refer to status, special skills, charisma, etc., that an actor may bring to an action situation. For example, human agents utilize power in interaction by drawing on facilities such as the ability to allocate material and human resources. In so doing they produce and reproduce structures of domination. Finally, human agents sanction their actions by drawing on rules (policy, etc.), norms and standards of morality and thus produce and reproduce social structures of legitimation. The three dimensions are inextricably interlinked, while signification is structured through language, language use always mediates aspects of domination and has a normative-force. Therefore, it is useless to analyze structures of signification without grasping their connections with domination and legitimation. The basic dynamics of structuration are summarized in Figure 2.

To complete this brief outline of structuration theory concepts, two further aspects need to be highlighted. First, there is no omnipotent agent who can forsee all effects of his or

\begin{tabular}{|c|c|c|c|}
\hline STRUCTURE & Signification & Domination & Legitimation \\
\hline MODALITY & $\begin{array}{l}\text { Interpretative } \\
\text { scheme }\end{array}$ & Facility & Norm \\
\hline INTERACTION & Communication & Power & Morality \\
\hline
\end{tabular}

Fig. 1. Analytical elements of the duality of structure. From Constitution of Society: Outline of the Theory of Structuration (p. 29) by Anthony Giddens, 1984, Oxford: Blackwell Publishers and Berkeley: University of California Press. Copyright 1984 by Anthony Giddens, Reprinted with Permission. 


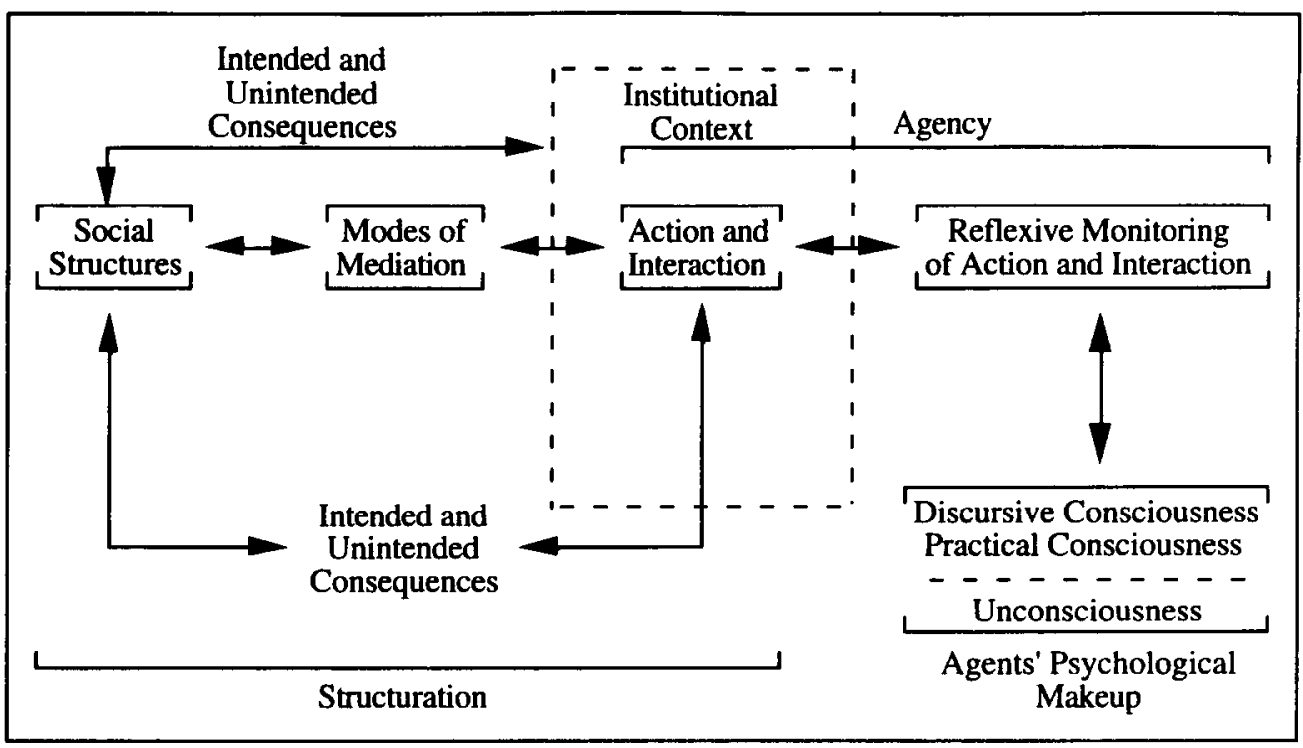

Fig. 2. The dynamics of structuration. Modified from "Structuration Theory in Management Accounting," by N.B. Macintosh and R.W. Scapens, 1990, Accounting, Organizations and Society 15 (5) p. 459, Pergamon Press Ltd.

her action. Structuration theory acknowledges that human action often has unintended and indirect effects on the larger institutional system within which the agents are situated. These effects may take place in any of the dimensions of social structuring: systems of meaning, power, or legitimation. Thus, the results of any purposeful conduct cannot be guaranteed. Second, it is essential to examine how social activity is distributed temporally and spatially in order to understand the conditions of its structuring. Time-space relations are not just "boundaries" to social activity, they are fundamental to the way that social activity is structured and "regionalized" in time and space. In this sense a social system is formed by intcractions that take place in time (historically) and space (geographically) and which are affected by the type and nature of resources deployed and rules invoked (structure). It is interesting to note that Holt (1988) seems to have understood this idea. He views coordination mechanisms that emphasize a spatial ordering of activities and various spatial metaphors as important to the design of CSCW applications (see also Stefik et al., 1987a, 1987b). Similar ideas of time relations are also emphasized by Kling (1987) in his web model of computing.

\section{A DEFINITION OF COOPERATIVE WORK}

Within the structuration theory framework, cooperative work may be defined as cooperative practices drawing upon specific rule/resource sets that are jointly produced and reproduced through shared, recurrent social and economic interactions among individuals. In cooperative practices, we are thus concerned with shared purposive activity that can only 
be established through "cooperative action." Examples of such practices can be found in large design projects that require numerous engineering specialists. On the other hand, hierarchical "programming" of social conduct through procedures in the sense which Galbraith (1977) defines it is not "cooperative work." Cooperative work is distinguishable from other types of work by its form and content, that is, its products, and spatial, time, and social distribution of tasks. It can be generally characterized by complex and intense interdependencies of activities, which are in turn dependent on the agents' shared understanding of the work process and the social context. In cooperative work the relationships among the agents are formed through planned structuring, deliberate discursive action, as opposed to spontaneous linking, such as in a market.

The nature of cooperative work can be further illustrated by the concepts of social and system integration (Giddens, 1984, p. 376-377). By social integration we mean the reciprocity of practices and social codes to which actors adhere when they are copresent in the work situation. Examples of social integration in CSCW are numerous, including participation modes, turntaking, group roles, specialized idioms and dialects, and even specialized humor associated with the use of the applications. System integration on the other hand deals with reciprocity practices and social codes among groups or collectivities across extended time-space, outside conditions of copresence. Two examples of this system integration phenomena are "weak ties" (Kiesler, 1986), and local bindings of commitments over time and organizational space (Winograd \& Flores, 1986). In general, cooperative work relies heavily on the reciprocity of practices among networks of agents, and routinization (the development of a vast reservoir of taken-for-granted activities) which enable frictionless encounters, effective and efficient social interaction. In most cases social and system integration takes place on the level of agents' practical consciousness and does not necessitate written codes or guidelines. This definition entails detailed and well-developed systems of signification and stocks of knowledge (interpretive schemes) on which the cooperative effort can rest. However, it does not set any specific requirements on power relations (such as selfdetermination or democratic management), nor on legitimation.

The notion of agency as included in structuration theory also holds implications for the analysis of cooperative work. One important implication is that cooperative work practices can be a vital means of meeting agents' unconscious needs for ontological security expressed on the unconscious level (see Figure 2). Such work practices can help to maintain social identity, achieve meaningful social interactions, and develop self-esteem and psychological security. On the level of practical consciousness, cooperative work involves development of finely grained and rich stocks of knowledge that sustain and provide rationalizations of agents' intensive cooperative encounters (such as meeting behavior). At the discursive level, the cooperative practices involve agents' capabilities to refine, discuss, and evaluate cooperative practices in order to transform them or to provide reasons for their existence in specific forms over time and space.

\section{A NORMATIVE DEFINITION OF CSCW}

In the literature the concept of computer support has been mainly approached from two different angles: technological and functional. The first limits the idea of support to the application of hardware and software that address the needs and requirements various in- 
dividuals have for "group work" (Greif, 1988). However, this sort of intuitive description does not provide any deeper understanding of the nature or types of support required. The second aims to define a taxonomy of tasks that form the integral part of group processes, and identifies a set of needs and problems that can be addressed by computer support (Huber, 1984; DeSanctis \& Gallupe, 1985). Functional analysis is applicable to defining requirements for situation specific CSCW applications, and in trying to improve their "positive" impact and rapid adoption in organizations. It, however, does not describe in any theoretical or conceptual way what computer support means and why a computer can enter as an important element into cooperative practices.

We propose a third angle, the ontologically focused approach, that conceptually characterizes the nature of computer support in cooperative practices and institutions. We believe that neglect of the careful delineation of the social nature (ontology) of the research object has led to too many one-sided analyses of technologies at the expense of understanding the social nature and process in which computers enter as focal elements of cooperative practices. From this perspective, we propose the following definition:

Computer Supported Cooperative Work applications are open evolutionary structures embedding organizational and linguistic rules and serving as resources that mediate and transform cooperative interactions via recurrent use-processes (procedures and practices) within specific organizational contexts.

Our definition clearly embodies fundamental ontological assumptions about CSCW that have been ignored in the past. We view CSCW applications as social structures and consider their use-processes as modalities that are shaped by the organizational context. We also believe that three basic characteristics distinguish CSCW applications from other computer-based applications. In the following we will explain in detail the notion of CSCW as social structures, and discuss the three distinguishing characteristics: (a) emergent properties, (b) use-processes, and (c) organizational contextuality.

\section{CSCW APPLICATIONS AS SOCIAL STRUCTURES}

We can now explore the notion of CSCW applications as social structures that constrain and are recursively reproduced by cooperative interactions established because of the actors' needs for integration within the organizational context. The reader will recall that structures consist of "generative rules and resources upon which actors draw" for the production and reproduction of cooperative work practices. From this perspective we view $\mathrm{CSCW}$ applications as rules and resources in support of cooperative work. Accordingly, the lcvel of support is definable by the scope of the activity in "cooperative work," the organizational context within which the social interactions are computer mediated, and the actors' capacities and involvement in the interactions.

CSCW applications act as resources to the extent that they embody means and material of labor. As a resource they provide a medium around which cooperatively organized work can be structured, and which mediates and transforms social interactions. Consider, for example, the use of computers in CAD/CAM applications. In most cases the application constitutes a means and a space of objects of work that affect the conditions and outcomes of social structuring. Computers embed rules to the extent they codify them in written form 


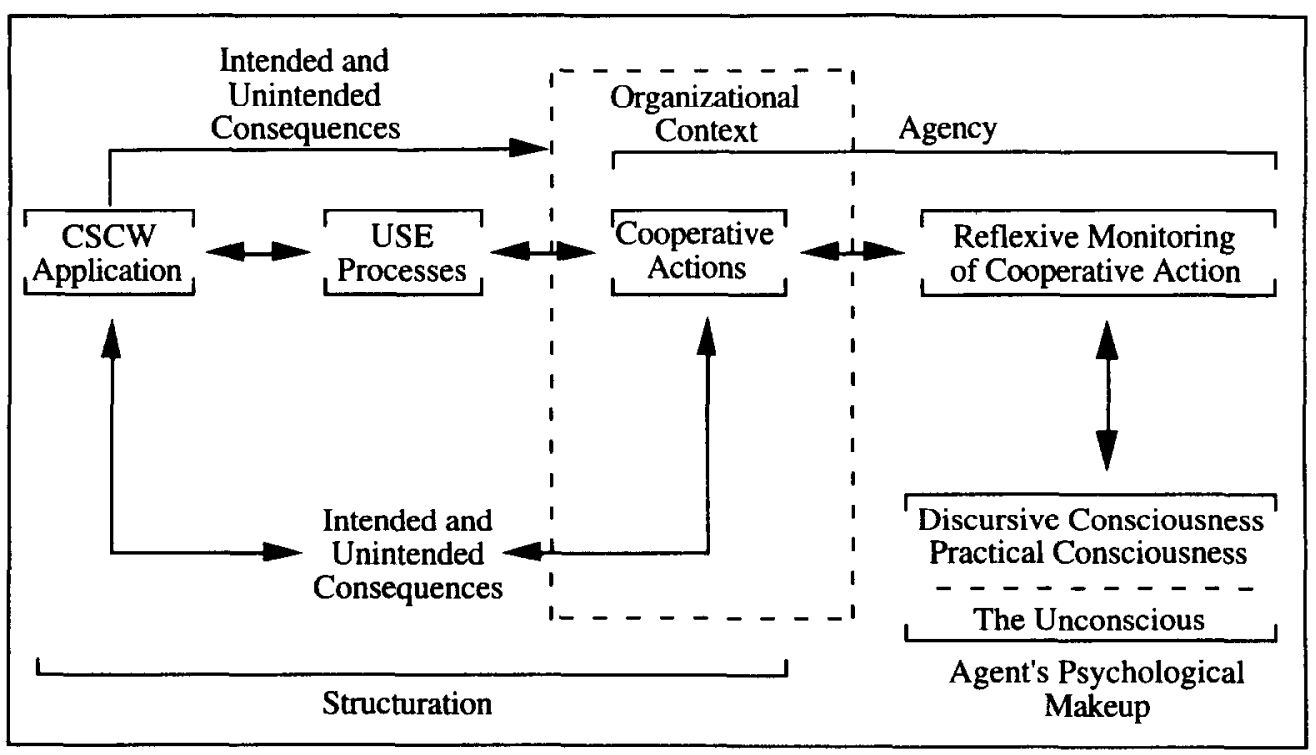

Fig. 3. Structuration dynamics of computer supported cooperative work.

or integrate rule-governed practices of computer use that define significant aspects of social conduct. This means that computers mediate and transform communication practices, organizational rules and norms, hierarchies, and role expectations. An example of computer-enforced rules would be communication patterns routinely followed in electronic mail that are governed, for example, by rules that: (a) define access to mail, (b) regulate how to maintain mailing lists, and (c) define acceptable protocols of communication behavior, etc. In this sense, these applications form one set of available rules and resources which agents can instantiate in their cooperative interactions. The division into two of modes of computer support: rules and resources, is not clear-cut in the sense that either the applications embed rules, or they serve as resources. Instead, as a mediating social structure any application entails the presence of both, and the division serves only to point out that wc can analytically search for different strategies in discerning and developing computer support. Accordingly, these applications provide a powerful and malleable means to shape work processes by crafting novel resources and enforcing new rules and protocols that can be drawn upon in social interactions.

It is important to note that our characterization of computer support in structurational terms implies also that agents' are skillful and knowledgeable actors in drawing upon CSCW applications to support their cooperative interactions (cf. Figure 3). This means several things. First, the applications can establish one means to secure the agent's ontological security by providing routine and regular interactions that are vital for satisfying such a need. Second, the concept of practical consciousness implies that agents know a great deal more than they can articulate about how and why they draw upon such applications in their interactions. Therefore, the applications very often exhibit a "seamless" integration into the agent's day-to-day routine and she is not necessarily aware of how and why she is using the 
application. Third, agents can discursively reason on the uses of the applications, for example, why and how they are drawn upon in social encounters. However, these rationalizations are often radically different from prior justifications to use and build the system given by its designers or sponsors. The reason for this is that designers and managers very rarely imagine or foresee cooperative practices that are made possible by computer mediation and how these practices are based upon specific structures of domination, legitimation, and signification (Grudin, 1990). Moreover, the transformative capability of computers and the impossibility to describe in detail agents' action make complete descriptions impossible.

\section{CHARACTERISTICS OF CSCW APPLICATIONS}

The fact that CSCW applications can enter as ruies and resources in a social space is itself nothing special; the same interpretation can be extended to any other computer application. It is our contention, however, that the three distinguishing characteristics of these applications; emergent properties, use-processes and organizational contextuality, identify them as an independent area of inquiry. Although it may be argued that all CSCW applications need not have these three characteristics, we view these as indicative of an ideal type application. This ideal type defines the scope of problems and solutions in which the design, delivery, and evaluation of these applications can be meaningfully exercised and enacted. It is important to note that several researchers have identified similar characteristics (cf. Sörgaard, 1988; Robinson, 1989).

\section{EMERGENT PROPERTIES}

Emergent properties are those features such as openness, evolutionary nature, and goal ambiguity, which support innovation and reinvention of cooperative work procedures and practices over time by instantiating different combinations of rules and resources, and creating new use-processes. In this sense the application can be viewed as duality of structure that enables, as well as constrains, cooperative interactions, and is also reproduced by them.

CSCW applications could be more effective if they display openness and do not aim at a procedural closure of practices. Workers in cooperative environments draw on stocks of knowledge for interpreting and coordinating each other's actions, which evade formal description (Ngwenyama, 1987). Accordingly rules underlying CSCW applications will often be tacit and informal. The concepts of practical and discursive consciousness explain this phenomenon; knowledge of rules and resources of intelligent actions exist as memory traces which are made manifest only in instances of action and interaction. There is also the issue of unintended consequences; the implementation of an application often leads to new possibilities that are not conceived in the original design rationale. Moreover, the user can always bypass the system either by deciding when a particular rule applies (Auramäki \& Leppänen, 1988), refining a new sct of rules, or ignoring the rules altogether (Flores, Graves, Hartfield, \& Winograd, 1988; Malone \& Kum-Yew, 1987). These features apply mostly in those applications that embed and invoke rules such as bulletin boards, coordination tools, and project management tools. 
The evolutionary nature means that $\mathrm{CSCW}$ applications could embody the means to reshape and redirect recurrent organizational practices which, in turn, reshape and redirect applications based on the duality of the structure. As users interact over time via these applications they will produce and reproduce structures of meaning, create and recreate new facilities to support emerging work patterns, and new norms and standards of cooperative work. The dynamic, often unconscious adaptation of CSCW applications into a larger ecology of organizational processes and practices can be explained by the evolutionary nature of these applications. This becomes clear when we observe how the applications link together with and become part of the repertoire of structures of signification, domination, and legitimation.

Another important characteristic is goal ambiguity. From a structuration theory perspective organizations have no goals or needs. Goals are ascribed to agents' action or their attempts to rationalize their action. Since agents can never describe their action completely due to its practical content, the goals are also always ambiguous and negotiable. This becomes very evident with applications targeted at seamless integration with cooperative work practices. It is unreasonable to expect that goals can be identified and well specified for such applications, they are situation dependent. The goals of every cooperative work situation are negotiated and renegotiated by the involved actors based on their own needs which change over time. Therefore, CSCW applications do not support clearly definable organizational goals, as do, for example, more traditional information systems such as inventory control or material-requirements planning systems (i.e., decreased inventory cost, faster turn-around times and more efficient inventory operations). In this regard, traditional goal-oriented approaches to justifying computer-based applications are often inappropriate for CSCW applications. Further, the high degree of embeddedness of these applications into work practices and the organizational context makes it difficult to identify quantifiable benefits. It is not surprising then that many studies cite qualitative responses such as "higher interaction," "better satisfaction," and "shorter meetings" to questions concerning benefits. Usually this applies specifically to generic "CSCW shells" such as electronic mail, bulletin boards, conferencing, or meeting technologies.

\section{USE-PROCESSES}

From the structuration perspective three use-processes can be defined: (a) collective, (b) autonomous, and (c) interpretive. Collective use means a CSCW application is expected to provide an arena for significant cooperative computer-mediated interactions among its users, that is, interactions that lead dynamically to changing how rules and resources enter into interactions and help to sustain reciprocity of codes and behaviors on which these interactions are based. All computer use is in some sense "collective." For example, the operation of an airline reservation system depends on the collective action of several operators. They are bound together through their shared knowledge and collective experience with the reservation system. However, this type of system is hardly conceived as a CSCW application, and its use-process is not collective in the sense defined here. The reason for this is that, from the application point of view, the system does not provide an arena for significant computer-mediated interactions among the operators. This characterization also suggests that users are likely to view collective use of CSCW applications both as mediums 
for, and products of meaningful social interactions. A good example of this is bulletin boards.

On the other hand, the interactions among users of a CSCW application vary in terms of their time-space coordinates and in terms of the number of user interactions involved. For example, interactions can be mediated in several time and space coordinates, such as in computer conferences, or they may be located in one time-space coordinate, such as meeting support (Johansen, 1988; Ellis et al., 1991). Accordingly, the collective use-process of these applications serves to increase the level of social and system integration and promotes high social visibility among its users. These phenomena have been observed in studies of organizations. Kiesler (1986) and Feldman (1987) found that applications, such as electronic mail, support new and improved modes of system integration called "weak ties," which were infeasible before electronic mediation. Likewise, Bannon and Schmidt (1990) observed increased social visibility of users involved in computer conferencing. High levels of social and system integration suggest that these applications support interactions characterized by a long time span, relational nature (in contrast to a discrete event), participants' incomplete expectations and uncertainty, and (sometimes) ambiguous, or shared values. This applies more specifically to applications that provide "generic" services to boost cooperative activity such as coauthoring systems, meeting support, or electronic mail. Specific and narrow services, such as calendar management or project management tools, instead seem to offer more tight local bindings of practices in limited time and space segments.

Autonomous use refers to the high degree of voluntary participation that characterizes CSCW applications. This is not usually the case in traditional information systems; usage is nearly always mandated by fiat or ipso facto the lack of options. In contrast, individuals can decide not to use CSCW applications. However, there is always strong social pressure and the lure of high social visibility that strongly motivates participation. Hence, system usage is often legitimized by cultural "ethos," symbolic value, group pressure or economic incentive, rather than by bureaucratic procedures (cf. critical mass theory, Markus, 1987). It is important to note however, that there are several situations where it is only possible to maintain large-scale computer-mediated interactions if system use is mandated (Markus \& Connolly 1990). This is especially true when the computer provides the resource (facility) on which a complicated work process is dependent, and all the users must use the system to smoothly coordinate and monitor their practices. Some examples are group decision making, electronic meetings, project management, etc. Nevertheless, from a structuration theory perspective we see that these applications cannot be legitimized by bureaucratic sanctions alone. We need look no further than the failures of many CSCW applications for evidence of this. The meaning of the application negotiated within the organizational context via the usc process plays a significant role in legitimation of it.

Interpretive mode of use implies that the meaning of data flows from the users' joint interpretive accomplishments. The semantics of data (or procedures) are not fixed beforehand and coded in a formal structure, such as a database schema. From a structuration theory perspective we would argue that the types of rules being invoked in CSCW applications could be described as: intensive, tacit, informal, and weakly sanctioned. They are partly embedded in the application and partly within a larger organizational context, and are continually evolving, being reinterpreted and renegotiated in action. Consequently, their meaning and modes of signification are reproduced by ongoing uses of the system. The following observation by Flores et al. (1988, p. 160) speaks to this issue: 
This is a key design issue: Let people interpret the natural language, and let the program deal with explicit declarations of structure (such as the user's declaration that this is a request). The conditions of the fulfillment rest in the interpretations of speaker and hearer, not in the structure of the text.

Robinson (1989) also supports this idea, he puts forward the notion of "double-level languages," and insists that the modes of signification and functionality offered by the CSCW application must be broadened by facilities that allow users to freely negotiate meaning attributions, task allocations, and articulation of the work process, in action.

\section{ORGANIZATIONAL CONTEXTUALITY}

The concept of contextuality emphasizes the visible role of the organizational context to $\mathrm{CSCW}$ applications. These applications are also media and products of cooperative interactions that are embedded in the larger organizational context. The structural properties of the organizational context enable, sustain, and redirect the uses of the system. Therefore, its delicate articulation and disclosure should encompass the analysis and definition of these applications (cf. Orlikowski, 1989). Inertia in the organizational context also explains the many obstacles to "implement" applications (Markus \& Connolly, 1990; Grudin, 1990). Focus on the context shifts our emphasis from the well-defined functions and structure of the application into ambiguous, and often contradictory, long-standing and deeply-seated practices that precede and affect the appropriation of CSCW technologies. For example, in the design of a meeting support system one must ask what social structures (power, legitimation, modes of signification) are reproduced in meetings? What social intentions they serve? And what seemingly irrational practices are served through meetings? Consequently, the outcomes of using the technology are largely dependent on the structural properties of the social system.

This aspect is illustrated in some discussions about the uses of CSCW applications. For example Foster and Stefik (1986, p. 14) noted: “Cognoter establishes a working framework both in the software and in the implicit rules of the game" (emphasis added). In effect, CSCW applications can shape attitudes and social processes. When tools like Cognoter become widely used, they may have an important effect on large organizations as carriers of problem-solving "culture" such as modes of signification, power distribution and so forth. Field studies on the use of Coordinator have clearly pointed out the importance of the organizational context to CSCW research and development. It has been shown that while Coordinator has had success in stable, hierarchical, authoritarian organizations in which rules of interaction are explicit and strictly enforced (Durham, 1988), it has failed in many organizations that are based on open, unrestricted, and negotiated interactions (Grantham \& Carasik, 1988). The main criticism is that the underlying paradigm of work of the software is not extensible enough to accommodate differences in the phenomenology of work supported by different organizational contexts (Robinson, 1990).

Further, the structuration process in any organizational context is always contradictory and inconsistent. Because the rules and resources available within the organizational context are generally not coherent and consistent, and their deployment does not necessarily lead to the same outcomes. Rules and resources often contradict each other; one set of rules may undermine or work against another, equally valid set. There also is no overarching 
logic of action that unfolds in agents' conduct. Individuals' choices are often random and constrained by their knowledge of the possibilities. CSCW applications are, thus, one of many inconsistent sets of organizational rules and resources that can be drawn upon. Further enforcing their usage (i.e., the rules and resources they constitute) may undermine the achievement of other important goals.

\section{IMPLICATIONS FOR RESEARCH AND DEVELOPMENT}

In the introduction we raised four questions that are fundamental to building a systematic agenda for CSCW research, namely: (a) What constitutes CSCW applications; what is the nature of cooperative work, and what do we mean by its computer support? (b) What is an appropriate theoretical framework for apprehending the intricate issues of the CSCW problem space? (c) What are the major research problems suggested by the framework? and (d) What research methods are appropriate for inquiring into the CSCW problem space?

We have examined the first two questions in much detail; in response to the first we have proposed definitions for cooperative work and CSCW applications. In response to the second question we have sketched out a structuration theory framework and demonstrated its usefulness for conceptual analysis by deriving our definitions from it. In the following we will address the questions of research problems and inquiry strategies.

From the above conceptual analysis we have identified seven research issues that need to be investigated. We are not suggesting this is an exhaustive list; it should be viewed as a starting point for building a coherent and comprehensive research agenda. For the sake of clarity we frame the issues as the following seven questions:

1. What features are important for CSCW platform software?

2. What methods are appropriate for $\mathrm{CSCW}$ research?

3. What systems development approaches are appropriate for efficient and effective CSCW application development?

4. What are appropriate features for a CSCW system development methodology?

5. How do CSCW use-processes and organizational structures shape each other over time?

6. How do specific CSCW applications enable and/or constrain specific work processes?

7. How do CSCW applications change the "parameters" of social and system integration and thus impact the systemic features of patterned social interactions?

Research Issue 1: A careful study of cooperative interaction and use-processes of CSCW applications is necessary to define the key features of platform software. More systematic empirical evidence needs to be gathered about the characteristics of use-processes and ways computer applications emerge and are instantiated by agents in their cooperative practices. The distinction into rules and resources suggested by the structurational account needs to be elaborated as to how rules and resources of legitimation, signification, and domination are inextricably interwoven in these applications. This evidence could be used to come up with a set of core features upon which software architectures and platforms could be based. It should be clear from the above discussion that the evolutionary nature of these applications argues for an open architecture in the platform software.

Research Issue 2: The structuration theory framework provides a basis for these empir- 
ical studies on the use of CSCW systems in organizations. A major contribution of this approach is the clarification of the interlinkages between use-processes with CSCW architecture and functionality. This enables, for example, the investigation of resistance to specific applications by examining structural contradictions and conflicts between software architecture and ongoing cooperative practices that sustain specific structures of domination and legitimation. We believe that the three characteristics of CSCW can be used as a basis to generate empirical studies that could focus on the features of the use-processes, the organizational context, and cooperative practices that they link together. Another important contribution is the theoretical basis for studying the processes of institutionalization of applications within organizations. The focal concepts of legitimation and routinization in the structuration process are important here. Because structuration theory can be regarded as a metatheory to order the inquiry into social life, it can also be used to justify methodological choices made. Structuration studies are open to several research strategies and modes as long as the research methods applied are suited for the chosen inquiry into social practices and are also sensitive to the meanings that the constituent actors ascribe to them; as all inquiries into social practices presume ethnography. Therefore, qualitative research techniques are integral to all types of inquiry. This means that detached observation and laboratory experimentation without useful theoretical and data triangulation would not yield much useful results, because CSCW applications as social structures can only be analyzed by examining the rules invoked and resources deployed by the involved actors in practical settings, and by recognizing the reciprocity of practices and social codes to which agents adhere. Keeping in mind the distinction of practical and discursive consciousness of the actors, the fact that the actors can do more than they can say, it is necessary for the researcher(s) to use several data gathering techniques that do not rely on self-reported data only.

Research Issues 3 and 4: Systems development methodologies for CSCW applications have received little attention in research. Our characterization of the CSCW applications as emergent systems that exhibit the practical consciousness of the agents and duality of the structure implies that no formal rigid methodology is enough or sufficient in developing applications. Because actors know more than they can say, and whatever they say is not necessarily the same as what they will do when they use the application in future. This suggests that any attempt to completely specify the rules and resources that underlie cooperative practices is doomed to fail. What is required is an open-ended emergent process that supports the transformation of rules, resources, and work processes? This point is clearly understood by Malone et al. (1988); they have designed an open architecture for the Object Lens that allows users to clearly define and redefine formal procedural rules and relationships over time.

In an open environment, application devclopment does not have to start from discovering formal, explicit rules that underlie tasks, and then gradually move to automate exceptional cases until all situations are formally described and transformed to algorithmic solutions. Instead, users are expected to freely decide which rules to draw upon and enforce. Usually, the application emerges from a set of "base rules" that instantiate a set of "basic" interactions. During their use the users are expected to negotiate new sets of rules and associated use patterns "on the fly." Such an emergent approach to CSCW application development does not mean that the process or its products will be completely unpredictable. As pointed out earlier structuration emerges from the agency/structure dynamic within the organizational context. In this regard, the products of an emergent approach to applica- 
tion development (evolution), will be characterized by the organization's structures of sig." nification, legitimation, and power. Furthermore, the directions application evolutions can be anticipated by carefully studying the resources and rules, and how they link to these organizational structures.

Designing and delivering a CSCW application involves an act of critically examining the possibilities of doing things in other ways and reflexively anticipating use situations in which specific rules and resources are instantiated. This helps to find misfits between practices and structures (or competing structures) which users draw upon, or misfits between agents' capacities and available resources. Thus, design episodes can help to anticipate possible failures of linking structures into practices or to transform practices by linking new structures into the organizational context. In this reflexive anticipation of socially-constituted practices, structuration theory offers ways to organize inquiries and to assemble debates of possible courses of action. In particular its idea that structures of legitimation, signification, and domination are inextricably linked and simultaneously present requires that methods (or rather modalities of design practices) recognize all these aspects of social structuration. So far, only issues of signification in CSCW development have been discussed in the literature (c.f., Winograd, 1987).

Research Issues 5,6, and 7 point out the need to analyze the impact of CSCW applications on the organizational context. Research issue 5 points out that applications and organizational structures are likely to shape each other over long periods of time. This evaluation is largely dependent on the dynamic processes of structuration, that is, the constitutive practices that reproduce structure and the social system. In this study it is important to note that the structuring of the technology is interactionistic in the sense that the structures shape cooperative practices which in turn shape the structures. There is no causal implication however, because agents can reflexively monitor their action and decide to act differently.

An important issue then, is how and why transformations in cooperative interactions take place when CSCW technologies are introduced into the work situation, and how these transformations affect the conditions and outcomes of its social structuring. Not surprisingly then, researchers in the field are interested in how work is transformed by computer technologies to cater for "cooperative" aspects of work, and what qualities the relationships between the work process and the computer system can and should acquire in such a setting. Therefore, the problem of how to construct software and hardware configurations that elegantly embody rules and resources, and which agents can successfully apply in cooperative settings, is of great significance to research. The structurational analysis of CSCW helps to characterize a broad range of common features that are typical to situations where computers support cooperative work. From this perspective we can elaborate how the relationships between a typical CSCW application and its user communities are dynamically constituted as modalities within the organizational context in which it is embedded. These relationships between agents (users) and their $\mathrm{CSCW}$ applications we defined as use processes (see Figure 3).

Structurational studies of CSCW applications should emphasize the dynamic interactions between cooperative work and its computer support within the larger organizational context. For example, a structurational study of electronic meetings of GDSS should go beyond traditional analysis of the decision situation (in terms of complexity and type) and what rules (protocols and participatory modes) and resources (such as meeting facilities) are drawn upon in group process. It should also explore how decision situations are de- 
fined, enacted, exercised, and reproduced within the larger organizational context and its modes of signification, legitimation, and power structures. In this way structurational analysis could offer well-founded theoretical guidance for examining changes in the basic premises of social structuring of $\mathrm{CSCW}$ and the work situation, and how these changes affect the prevailing social order. In addition it could give some insight into the role of organizational context with regard to the successful implementation and adaptation of CSCW technologies.

While CSCW applications embed rules, serve as a resources, and thereby enable, modify, and sustain "cooperative" practices, they are at the same time shaped by existing institutionalized practices. CSCW applications reflect the history of organizational practices through which they are built and used. Therefore any analysis of CSCW cannot ignore the institutionalized nature of processes that serve to facilitate, as well as constrain, the use and development of CSCW applications. In other words, CSCW technology serves to reinforce those cooperative practices (and their modes of legitimation, signification, and power structures) that it embodies, and from which it has grown (for example, as meeting practices, work group norms along with the resources and rules drawn upon in these encounters). The organizational context must be recognized as integral to the design and evolution of any CSCW application. And organizational analysis must focus on describing and understanding those persistent social structures that constrain and enable cooperative work. CSCW technologies by themselves do not deterministically affect social structures. It is their design, deployment, and institutionalization within the particular organizational context, as well as their ongoing uses by particular users that together determines their impact on the social structure.

Research issue 6 points out that structures should be viewed as enabling and constraining cooperative practices; they do not merely constrain, they also make them possible - a point that functionalistic analyses often ignore. Point 7 is probably the most subtle issue in researching CSCW from a structurational standpoint. Giddens' own analysis usually focuses on mediating technologies that achieve higher levels of either system or social integration. In fact, he does not discuss many technologies of social integration, though such simple facilities as blackboards or physical arrangements of meeting rooms serve these "purposes," and he probably is aware of them. His primary focus has been on technologies that facilitate system integration (writing, telephone, transportation facilities). The analysis of available CSCW technologies shows that their potential in mediating social interactions is much more varied and flexible. Through the evolution of CSCW applications, we believe we can see an emergence of technologies that form a continuum from technologies embedded in situations of high social integration (such as meeting rooms) to technologies that mediate only system integration (electronic mail). "Middle-range" technologies include real-time multiuser editors, virtual meeting rooms, conferences, etc. It is likely that such technologies will shape the structuring parameters of social systems in ways that are largely unforeseen and unpredictable. Predictions of these developments in IS research most often portray optimistic ideas of "team based" organizations (see e.g., Applegate, Cash, \& Mills, 1988). Such analyses emphasize the positive and good "impacts" of CSCW applications on the social system. Based on our analysis of structuration theory we cannot concur on such predictions, as they ignore the domination and legitimation aspects that are always central in social structuring. What the likely impacts of CSCW applications in different institutional contexts are will be an interesting research challenge to examine and will help to test the viability of the structurational account of $\mathrm{CSCW}$. 
Acknowledgement-For lively and useful discussions, Esa Auramakt. Liam Bannon and Mike Robinson provided useful (but controversial) comments. Thanks to Dick Boland for his patient criticism and the three reviewers for their constructive comments.

\section{REFERENCES}

Applegatc, L., Cash, J., Mills, D.Q. (1988). Information tochnology and tomorrow's manager. Iharvard Businexs Review, November-December, $128-136$.

Auramäki, E* \& Leppänen, M. (1988). Exceptions and office information systems. In B. Pemici \& A. VerrintStuart (Eds.), Offec dexign methodologies. Amsterdam: North Holland.

Banon, L. Schmit, K. (1990), CSCW, or what's in a name. (Unpublshed manuscript), Aarhus University, Department of Computer Science, Aarhus.

Barley, S. (1986). Technology as an occasion for structuring: Observations of $\mathrm{CT}$ scanners and the social order of radiology departments, Administrative Science Quarterly, 31,78-108,

Boland, R.J., Jr. (1985). Accounting and the production of culture: Budgets and the process of structuration. Workshop on Accounting and Culture, European Institute of Advanct Management, Amsterdam.

DeSanctis, $G$., \& Gallupe, R.B. (1985). Group decision support systems: A new frontier. Data Base, 16(1), 377-387.

Durham, T. (1988). Organizational dinosaurs take on a human face. Computing. London: VNV Business Publications.

Ellis, C.A., Gibbs, S.I., \& Rein, O.L. (1991). Groupware-Some issues and experiences. Communications of the ACM, $34(1), 39-58$.

Feldman, M., \& March, J, (1981). Information in organizations as signal and symbol. Administrative Science Qutaterty, 26(2), 171-186.

Feluman, M. (1987). Electronic mall and weak hes in organizations, Offed Trothology and People, 31), 83-101.

Fores, F., Graves, M., Hartield, B., Winograd, T. (1988). Computer systems and the design of organizational interacton. ACM Trantactions on Office information Systems, 6(2), 153-172.

Foster, G. \& Stefik, M. (1986). Cognoter, theory and practice of collaborative tool. In Proceedings of the CSCW $86(\mathrm{pp}, 7-15)$.

Galbraith, J. (1977). Organization design. Reading, MA: Addison-Wesley.

Giddens, A. (1976). New rules of sociological method. London: Hutchinson Company.

Giddens, A. (1979). Centrat probletns in wocial theory: Action, strutum and contradiction in social antlysis. Berkeley, CA: University of California Press.

Giddens, A. (1984). The constitution of society. Cambridge, MA: The Polity Press.

Grantham, C.F. \& Casasile, R.P. (1988). The phenomenology of computer supported co-operative work. Inter personal Software. Berkeley, CA.

Greif, I. (Ed). (1988). Computer supported cooperative work: A book of readings. San Mateo, CA: Morgan Kaufmann Publishers.

Gndin, I. (1988). Why CSCW applications fal: Problems in the designs and evahation of organizational inter* faces. Procedings of CSCW' $88,85-93$.

Grudin, I. (1990). Groupware and cooperative work: Problems and prospects. In B. Laurel (Ed.). The Art of HWw man-Computer Interface Design. Reading, MA: Addison-Wesley.

Han, C.K., Walsham, G. (1989). Public policy and information systems in government: A mixed level anatysis of computerization. (Maragement Studies Research Paper 3/89): Cambridge University.

Holt, A.W. (1988). Diplans: A new language for the study and implementation of coordination. ACM Trmogetions on Office Information Systems, $6(2), 109-125$.

Howard, R. (1987). System design and social responsibility: The political implications of "computer supported cooperative work." Office: Technology and People, 3(2), 175-1987.

Huber, G.P. (1984). Issues in the design of group decision support systems. MIS Qtarterly, 8(3), $195-204$.

Johansen, R. (1988), Groupware computer support for business teams. New York: Free Press.

Kiesler, S. (1986). The hidden messages in computer networks. Harvard Business Review, $7,46-60$.

King, R. (1987). Defining the boundaries of computer across complex organizations. In R.J. Boland R.A. Hirscheim (Eds.), Criticat Issues in Information Sysems Research (pp. 307-362), Chithester: Iohn Wiley \& Sons. 
Kraemer, K.L., \& King, J.L. (1988). Computer-based systems for cooperative work and group decision making. Computing Surveys, 20(2), 115-146.

Lyytinen, K. (1991). Computer Supported Cooperative Work: Issues and Challenges. Unpublished manuscript.

Lyytinen, K., \& Hirschheim, R.A. (1987). Information system failures: A survey and classification of empirical literature. Oxford Surveys in Information Technology, 4, 257-309.

Macintosh, N.B., \& Scapens, R.W. (1990). Structuration theory in management accounting. Accounting, Organization and Society, 15(5), 455-477.

Malone, T.W., \& Kum-Yew, L. (1988). Object lens: A "spreadsheet" for cooperative work. Proceedings of CSCW '88, 115-124.

Markus, L.M. (1987). Toward a critical mass theory of interactive med universal access, independence, and diffusion. (Information Systems Working Paper \#9-87). Los Angeles: University of California, Graduate School of Management.

Markus, L.M., \& Connolly, T. (1990). Why CSCW applications fail: Problems in the adoption of interdependent work tools. In Proceedings CSCW'90 Conference on Computer Supported Cooperative Work. New York: ACM.

Mohrman, A., \& Lawler, E. (1984). Is technology and organizations-A review of theory and practice. In W.F. McFarlan (Ed.), The Information Systems Research Challenge (pp. 135-164). Cambridge: Harvard Business School Press.

Ngwenyama, O.K. (1987). Fundamental issues of knowledge acquisition: Toward a human action perspective of knowledge systems. Unpublished doctoral dissertation, Thomas J. Watson School of Engineering, State University of New York, Binghamton.

Orlikowski, W.J. (1988). CASE Tools and the IS Workplace: Some Findings from Empirical Research. In Pro ceedings of the 1988 SIGCPR Conference on the Management of Information Systems Personnel, New York: ACM.

Orlikowski, W.J. (1989). Division among the ranks: The social implications of CASE tools for system develop ers. In J. I. DeGross, J. Henderson, and B. B. Konnsynski (Eds.), Proceedings of the 10th International Conference on Information Systems (pp. 199-210). New York: ACM.

Orlikowksi, W.J. (1990). The duality of technology: Rethinking the concept of technology in organizations. (Working Paper 3141). Cambridge: Massachusetts Institute of Technology, Sloan School of Management.

Poole, M.S., \& DeSanctis, G. (1989a). Understanding the use of group decision support systems: The theory of adaptive structuration. In C. Steinfield and J. Fulk (Eds.), Perspectives on Organizations and New Information Technology. Beverly Hills, CA: Sage.

Poole, M.S., \& DeSanctis, G. (1989). Use of group decision support systems as an appropriation process. In Proceedings of the 22nd Annual Hawail International Conference on Systems Sciences (pp. 149-157). New York: ACM.

Riley, P. (1983). A structurationist account of political culture. Administrative Science Quarterly, 28(3), 414-437.

Robey, D., \& Zmud, R. (1990). Research on end-user computing: Theoretical perspectives from organization theory. In K. M. Kaiser and H. J. Oppelland (Eds.), Desktop Information Technology (pp. 15-36). Amsterdam: North Holland.

Robinson, M. (1989). Double level languages and co-operative working. AI Society, 5, 54-60.

Sheil, B. (1983). Coping with complexity. Office: Technology and People, I(1).

Sörgaard, P. (1988). A discussion of computer supported cooperative work. Unpublished doctoral dissertation, Computer Science Denartment, Aarbus University, Aarhus, Denmark.

Stefik, M., Bobrow, D.G., Foster, G., Lanning, S., \& Tatar, D. (1987a). WYSIWIS revised: Early experiences with multiuser interfaces. ACM Transactions on Office Information Systems, 5(2), 147-167.

Stefik, M., Foster, G., Bobrow, D. B., Kahn, K., Lanning, S. \& Suchman, I.. (1987b). Beyond the chalkboard: Computer support for collaboration and problem solving in meetings. Communications of the $A C M, 30(1)$, $32-47$.

Suchman, L. (1989). Notes on computer support for cooperative work. Unpublished manuscript, University of Jyvaskyla, WP-11, Department of Computer Science, Jyvaskyla, Finland.

Walsham, G. \& Han, C.K. (1990). Structuration theory and information systems research. Proceedings of the International Conference on Information Systems (53-59). Copenhagen: ACM Press.

Winograd, T. \& Flores, F. (1986). Understanding computers and cognition: A new foundation for design. Norwood, NJ: Ablex.

Winograd, T. (1987). A language/action perspective on the design of cooperative work. (STAN-CS-87-1158). Stanford: Stanford University, Department of Computer Science. 